\title{
Mid-Regional Proadrenomedullin as a Prognosis Biomarker of Mortality in Patients Treated with Non-Invasive Ventilation in the Emergency Room
}

Manuel Piñero Zapata ${ }^{1 *}$, Miriam Martinez Villanueva ${ }^{2}$, Francisco $V$ Aviles Plaza $^{3}$, Cesar Cinesi Gomez ${ }^{4}$, Aurelio Luna Maldonado 5 and Maria E Legaz Moreno ${ }^{6}$

${ }^{1}$ Faculty of Nursing, San Antonio Catholic University (UCAM), Spain

2Pharmaceutical Specialist in Clinical Biochemistry, University General Hospital "Virgen de la Arrixaca", Spain

${ }^{3}$ Medical Doctor Specialist in Clinical Biochemistry, Biochemistry Laboratory of the University General Hospital "Virgen de la Arrixaca" in Murcia, Spain

${ }^{4}$ Medical Doctor Specialist in Family and Community Medicine, Emergency Department of the University General Hospital "Reina Sofia" in Murcia, Spain

${ }^{5}$ Medical doctor, Murcia University in Murcia, Spain

${ }^{6}$ University Degree in Nursing, Emergency Department of the University General Hospital "Reina Sofia" in Murcia, Spain

*Corresponding author: Manuel Piñero Zapata, Faculty of Nursing, San Antonio Catholic University of Murcia (UCAM), Campus de los Jerónimos, №135 Guadalupe 30107 (Murcia), Spain, E-mail: mpinero@ucam.edu

\begin{abstract}
The mid-regional fragment of the peptide complex Proadrenomedullin (MR-proADM) is a ubiquitous biomarker tissue distribution in the human body with increased levels in the course of certain diseases of great importance for vital engagement and whose clinical course is often associated with dyspnoea.

Prospective observational analytical survey conducted on a group of 191 selected patients requiring urgent treatment with NIV due to acute or exacerbated chronic dyspnoea from any cause, except surgical or neuromuscular, between November 2012 and December 2013, to which they were, extracted a blood sample at the time of initiation of therapy with NIV to determine the MR-proADM.

The first aim of our study was to establish the association between plasma levels of MR-proADM and in-hospital mortality in patients undergoing NIV for acute respiratory failure regardless of source.

The median age of patients was 79-years-old and overall mortality by $22 \%$. Mid-regional pro-adrenomedullin presented a median of $0.57 \mathrm{nmol} / \mathrm{L}$ (from 0.29 to $3.19 \mathrm{nmol} / \mathrm{L}$ ), and the same amount was obtained for both survivors to deceased.
\end{abstract}

In our survey, the MR-proADM has not shown the ability to forecast for 30-day mortality of patients requiring treatment with NIV for acute respiratory failure, regardless of the precipitating pathology.

\section{Keywords}

Proadrenomedullin, Respiratory insufficiency, Respiratory failure, Non invasive ventilation, NIV

\section{Background}

The use of biomarkers is an essential accessory for the accuracy of diagnosis, the initiation of treatment, and the management of critical patients [1].

Kitamura published in 1993 the discovery of Adrenomedullin (ADM) [2]. ADM is a peptide which has an homology with the peptide related to the calcitonin and amylin gene, which all belong to the same family. These features are essential for its biological activity $[3,4]$.

A reliable quantification of ADM in plasma is difficult because of its short half-life, due to the fact that it varies its values in a short period of time [5]. However, a por-

Citation: Zapata MP, Villanueva MM, Plaza FVA, Gomez CC, Maldonado AL, et al. (2017) Mid-Regional Proadrenomedullin as a Prognosis Biomarker of Mortality in Patients Treated with Non-Invasive Ventilation in the Emergency Room. Int J Crit Care Emerg Med 3:024. doi.org/10.23937/2474-3674/1510024 Received: May 08, 2016: Accepted: May 09, 2017: Published: May 11, 2017

Copyright: (C) 2017 Zapata MP, et al. This is an open-access article distributed under the terms of the Creative Commons Attribution License, which permits unrestricted use, distribution, and reproduction in any medium, provided the original author and source are credited. 
tion of the native protein, the mid-regional fragment of the peptide complex Proadrenomedullin (MR-proADM), has been identified being more stable than the active molecule itself, whose synthesis is stoichiometrically related, and is secreted in equimolar amounts to the ADM [6], which indirectly allows its quantification [7].

Hypoxia stimulates the secretion of ADM [3]. The several effects, such as neuro-hormonal compensatory channel on target organs, gives it its wide biological activity, it is suggested that ADM acts as a powerful protective factor for the organism in situations of cellular stress, using mechanism diuresis and natriuresis, or contributing with the fight against infection, among others $[4,8]$. The relevance of the ADM is based on plasma concentrations of MR-proADM in humans, it has been shown to be increased in the course of major life-threatening diseases (sepsis, pneumonia, chronic obstructive pulmonary disease, myocardial infarction and heart failure), combining as counter-regulatory mechanism [9], and being very useful in risk stratification as it has been able to predict mortality in patients with these critical processes [5,10-15].

Patients with secondary dyspnea towards Respiratory infections, Aggravated chronic obstructive pulmonary disease, Acute Cardiogenic Pulmonary Edema (ACPE), Shock, etc., pathologies that cause or may cause, directly or indirectly, Acute Respiratory Failure (ARF), are in many occasions treated with Non-Invasive Ventilation (NIV) [16] as supportive therapy in the emergency room due to severity of the process. In various studies published on the MR-proADM, it has been found that patients suffering from the above described symptoms have high MR-proADM having been postulated as a prognosis of mortality.

The primary aim of our survey was to establish the association between plasma concentrations of the biomarker MR-proADM and 30-day mortality of patients which require to be treated with NIV in the Emergency Room (ER), regardless of the precipitating pathology. As a secondary objective, we purposed associate any other indicators of the study with 30-day mortality of these patients.

\section{Methods}

Prospective observational analytical survey carried out in the ER of University General Hospital Reina Sofia in Murcia (Spain) from November 2012 to December 2013 without any therapeutic implication on participants.

The survey followed the laws and regulations and was evaluated by the Clinical Research Ethics Committee C.R.E.C. The treatment, communication and transfer of personal data of the participants were in line to the Organic Act 15/1999 of December 13 on the protection of personal data.
Inclusion criteria: elderly patients who turned to ER due to acute respiratory failure of any etiology and needed urgent therapy with NIV. The inclusion of patients in the research was dynamic and consecutive.

\section{Exclusion criteria}

Patients under 18 , patients who were on dialysis, those whose admission was caused by surgical or traumatic pathology and those who could be in agony or pre-mortem at the time of the NIV set up.

\section{Sample collection and analysis}

At the beginning of the treatment with NIV, several blood tests were taken for arterial blood gas, blood count, biochemistry and coagulation; plus a blood sample was extracted in an EDTA plasma tube that would be used to determine the MR-proADM. After extraction, the samples were processed immediately; however, the sample for MR-proADM determination was centrifuged at 3,500 rpm for 10 minutes separating the plasma fraction and distributed into aliquots which were frozen at $-80^{\circ} \mathrm{C}$ to be analyzed once the rest of the samples had been collected.

The quantification of MR-proADM was conducted by the ELISA technique (Enzyme-Linked Immuno Sorbent Assay); a Human mid-regional pro-adrenomedullin, MR-ProADM ELISA Kit from "SHANGHAI YEHUA Biological Technology Co., Ltd.", Shanghai, China was used; this kit uses a double antibody Biotin sandwich technology, whose intra-assay accuracy has a Coefficient Oscillation (CO) under $10 \%$ and inter-assay under $12 \%$; measuring range is between 0.02 to $6 \mathrm{nmol} / \mathrm{L}$ and sensitivity is $0.011 \mathrm{nmol} / \mathrm{L}$.

\section{Data collect}

The general diagnose and analytical data were obtained from the clinical record, which had been transferred after having been determined; finally, the relating survival data was obtained from the files once they were reflected or directly from their families by telephone survey when we could not get the information any other way.

The variable "30-day Mortality" was the only dependent in the survey which took the values "YES" and "NO" including the positive value for all the patients deceased within 30 days of their admission to the ER, consigning the date of death for further statistical treatment.

The variable "Diagnose" corresponds to the consigned on the hospital discharge clinical report from the ER, the diagnostic "Respiratory Failure (RF)" refers to the one with pulmonary origin, mainly aggravated COPD; likewise, the diagnostic "Congestive Heart Failure (CHF)" which corresponds mainly to the ACPE.

\section{Statistical analysis}

For statistic calculations the program "IBM SPSS Statistics" v-19 was used. 
The categorical variables were described by their absolute values and percentages; and the continuous by their mean, typical deviations and confidence intervals of $95 \%$ (Cl 95\%) and/or median followed by the interquartile range and its $\mathrm{Cl} 95 \%$ in case the variable did not follow a normal distribution. Each one of the categorical variables were analyzed for the survivor and non-survivor group; checking the type of quantitative variable distribution by the Kolmogorov-Smirnov test.
The differences between the categorical variables were established on the "ji-square" or the Fisher's $F$-test; and for the quantitative the Student's $t$-test or the Mann-Whitney's $U$-test; what is more the relative risks were calculated with its $\mathrm{Cl} 95 \%$. To find out if there was an association between the quantitative variables and the different groups (quartiles of the MR-proADM) the ANOVA or Kruskal-Wallis test was used.

\section{Cases reported}

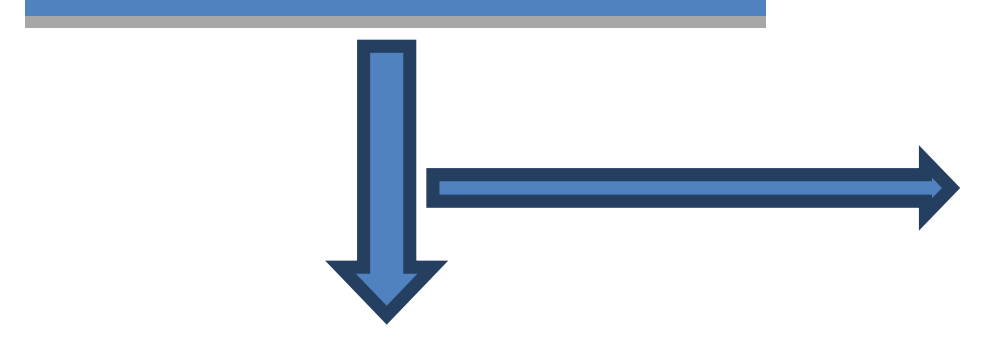

\section{2 cases of patients withCRF in dialysis}

\section{Cases}
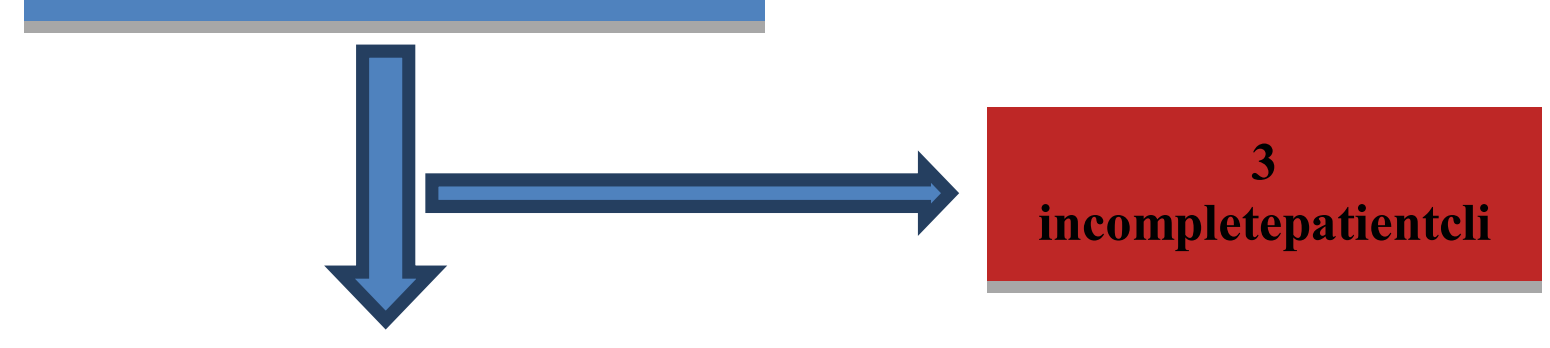

\section{Cases}

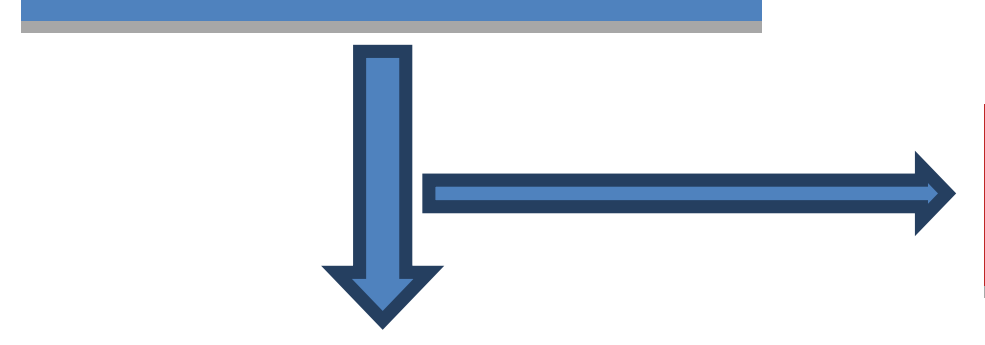

\section{4 duplicate cases in theselection}

\section{Cases}
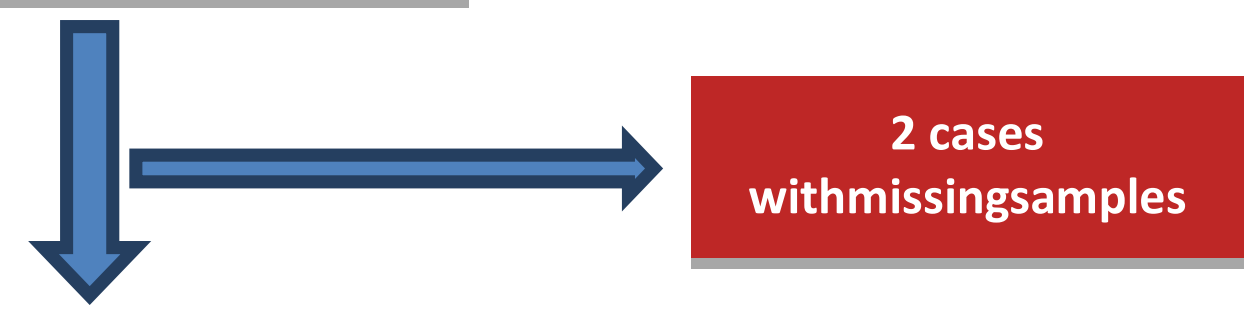

\section{Studied 191 cases of ARF treated with NIV}

Figure 1: Flow diagram for selection of cases. 


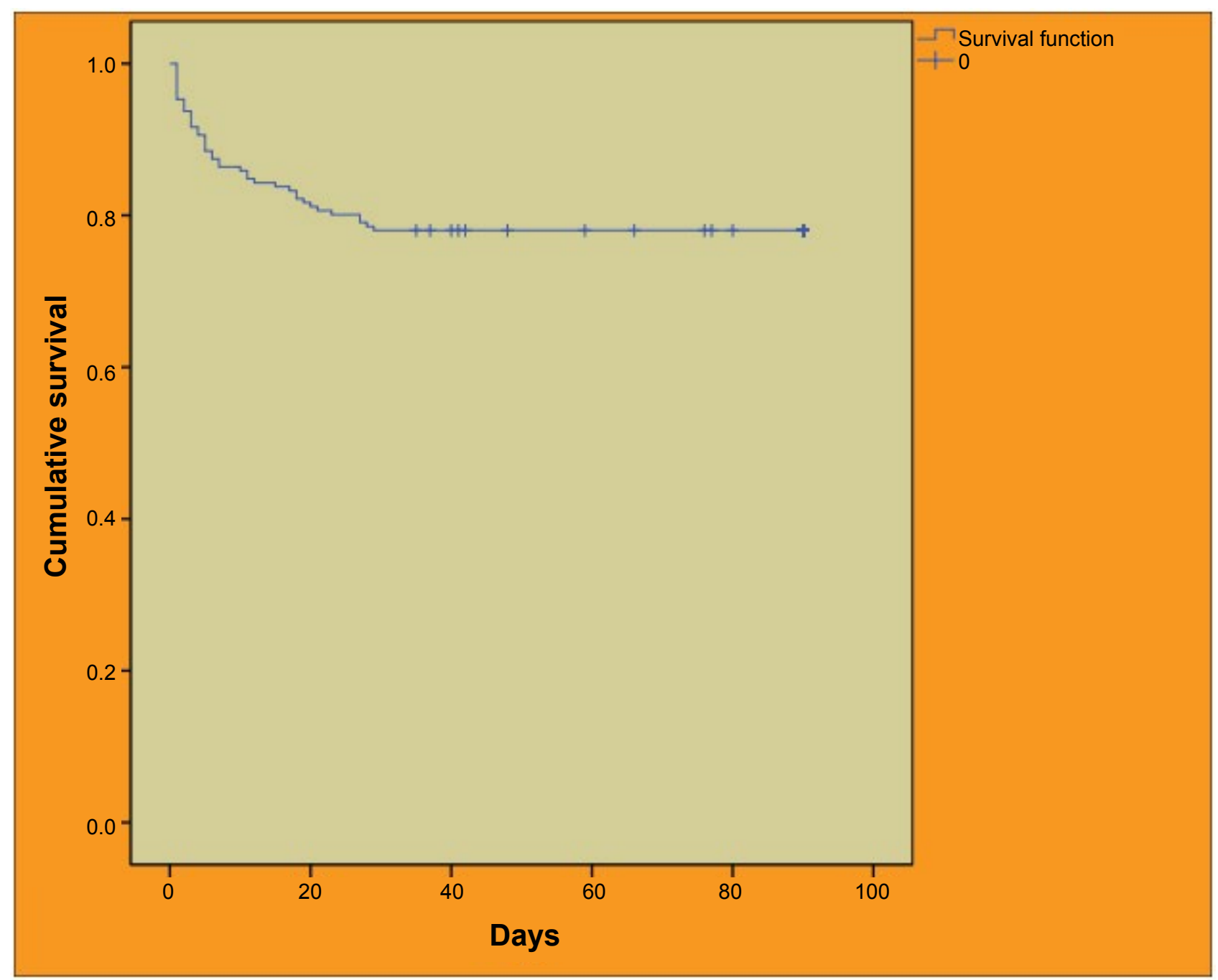

Figure 2: Survival Kaplan-Meier curve.

To discriminate the potential confusion of the variables in which association was found, a Cox regression was made in which it was introduced, and further including to the model the main variable of the survey, the MR-proADM.

ROC curves were used to search the discriminative power of the target biomarker for 30-day mortality, calculating the area under the curve.

The level of signification accepted was of $p<0.05$.

\section{Sample size calculation}

We considered an $80 \%$ potency, a type I error of 0.05 and a cut-off point of $1.5 \mathrm{nmol} / \mathrm{L}$ for the MR-proADM, as well as a rate of adverse events in the control group of $13 \%$ and $30 \%$ in the case group; to this $15 \%$ of estimation of losses was added, resulting a sample size of 202 cases.

\section{Results}

202 patients were valued being included 191 cases. The losses (5.44\%) were due to inappropriate inclusion ( 2 cases), incomplete patient clinical record (3 cases), duplicated ( 4 cases) and sample loss ( 2 cases) (Figure 1).

The age was between 24 and 96-years-old, average of 79 -years-old, $51.3 \%$ were males ( 76 for men and 81 for women); $61.78 \%$ exceeded 75 -years-old (57.6\% women). The deceased within 30 days since their admittance were 42 (22\%) (Figure 2), 21 men (21.4\%) and 21 women $(22.6 \%)(p>0.05)$.

The values of MR-proADM were between 0.29 and $3.19 \mathrm{nmol} / \mathrm{L}$, mean of $0.67 \pm 0.37 \mathrm{nmol} / \mathrm{L}$; they were not normally distributed with a median for the global population of $0.57 \mathrm{nmol} / \mathrm{L}(I Q R=0.22)$ and did not show significant statistical differences $(p>0.05)$ between the two groups of global population, survivors and deceased, therefore it was not possible to obtain an optimal cut-off point; the figures in the survivors group were $0.57 \mathrm{nmol} / \mathrm{L}(\mathrm{IQR}=0.26)$ and also $0.57 \mathrm{nmol} / \mathrm{L}(\mathrm{IQR}=$ $0.14)$ in the deceased. No significant statistical differences were shown in the MR-proADM between survivors and deceased at sample stratification by gender with an $A U C=0.451$ (Cl 95\%: 0.312-0.590, $p=0.499)$ for females and $A U C=0.512$ ( $\mathrm{Cl}$ 95\%: 0.381-0.664, $p=0.863$ ) for males, nor by discharge diagnose from the ER with AUC $=0.345$ (Cl 95\%: 0.213-0.477, p = 0.643) for CHF and with AUC $=0.526$ (Cl 95\%: 0.372-0.679, $p=0.526$ ) for RF from pulmonary origin.

The general characteristics of the patients can be 
Table 1: General characteristics of the patients. Comparison between survivors and deceased within 30 days from emergency room admission. Qualitative variables.

\begin{tabular}{|c|c|c|c|c|c|c|}
\hline & All cases & Survivors & Deceased & & & \\
\hline Variables & n (\%) & n (\%) & n (\%) & $P$ value & $\mathbf{R R}$ & C.I. $95 \%$ \\
\hline \multicolumn{7}{|c|}{ Personal characteristics } \\
\hline Age > 75 a. & 118/191 (61.8) & $82 / 149(55)$ & $36 / 42(85.7)$ & $<0.0001$ & 3.712 & $1.645-8.373$ \\
\hline Gender $=$ female & 93/191 (48.7) & $72 / 149(48.3)$ & $21 / 42(50)$ & ns & 1.054 & $0.618-1.798$ \\
\hline $\mathrm{BMI}>30$ & $82 / 173(47.4)$ & $68 / 139(48.9)$ & $14 / 34(41.2)$ & ns & 0.777 & $0.420-1.436$ \\
\hline Smoking & 97/187 (50.8) & $81 / 146(55.5)$ & $16 / 41(39)$ & ns & 0.594 & $0.340-1.038$ \\
\hline \multicolumn{7}{|l|}{ Personal history } \\
\hline HBP & 166/191 (86.9) & $127 / 149(85.2)$ & $39 / 42(92.9)$ & ns & 1.958 & $0.654-5.861$ \\
\hline DM & 93/191 (48.7) & $77 / 149(51.7)$ & $16 / 42(38.1)$ & ns & 0.648 & $0.373-1.129$ \\
\hline COPD & 76/191 (39.8) & $60 / 149(40.3)$ & $16 / 42(38.1)$ & ns & 0.931 & $0.537-1.616$ \\
\hline FEV1 < 50\% & 45/79 (23.6) & $37 / 65$ (56.9) & $8 / 14(57.1)$ & ns & 1.007 & $0.386-2.632$ \\
\hline Hypov. Syndr. & $41 / 190(21.5)$ & $33 / 148(22.3)$ & $8 / 42(19)$ & ns & 0.855 & $0.430-1.702$ \\
\hline Heart diseases & $125 / 190(65.4)$ & $98 / 149(65.8)$ & $27 / 41(65.9)$ & ns & 1.003 & $0.566-1.776$ \\
\hline Arrhythmias & $88 / 190(46.1)$ & $65 / 148(43.9)$ & $23 / 42(54.8)$ & ns & 1.403 & $0.820-2.399$ \\
\hline LTOT & 64/191 (33.5) & $47 / 149(31.5)$ & $17 / 42(40.5)$ & ns & 1.349 & $0.788-2.311$ \\
\hline Domiciliary NIV & $47 / 191(24.6)$ & $41 / 149(27.5)$ & $6 / 42(14.3)$ & ns & 0.511 & $0.230-1.135$ \\
\hline \multicolumn{7}{|c|}{ Laboratory variables } \\
\hline $\mathrm{Hb}<12$ & 78/191 (40.8) & $62 / 149(41.6)$ & $16 / 42(38.1)$ & ns & 0.82 & $0.513-1.548$ \\
\hline Creat $>1.5$ & $81 / 191(42.4)$ & $62 / 149(41.6)$ & $19 / 42(45.2)$ & ns & 1.122 & $0.657-1.917$ \\
\hline Bicarb $<22$ & 56/191 (29.3) & $40 / 149(26.8)$ & $16 / 42(38.1)$ & ns & 1.484 & $0.865-2.544$ \\
\hline Lact $>2$ & 76/182 (39.8) & $54 / 143(37.8)$ & $22 / 39(56.4)$ & 0.036 & 1.805 & $1.031-3.160$ \\
\hline \multicolumn{7}{|c|}{ ER discharge diagnoses } \\
\hline $\mathrm{CHF}$ & 88/191 (46.1) & $70 / 149(47)$ & $18 / 42(42.9)$ & ns & 0.878 & $0.511-1.508$ \\
\hline $\mathrm{RF}$ & $82 / 191$ (42.9) & $67 / 149(45)$ & $15 / 42(35.7)$ & ns & 0.738 & $0.421-1.296$ \\
\hline Sepsis & 6/191 (3.1) & $1 / 149(0.7)$ & $5 / 42(11.9)$ & $<0.001$ & 4.167 & $2.632-6.597$ \\
\hline Pneumonia & 7/191 (3.7) & $6 / 149(4)$ & $1 / 42(2.4)$ & ns & 0.641 & $0.807-1.507$ \\
\hline Others & $8 / 191(4.2)$ & $5 / 149(3.4)$ & $3 / 42(7.1)$ & ns & 1.17 & $0.689-4.491$ \\
\hline \multicolumn{7}{|c|}{ ER discharge destination } \\
\hline Hospitaliz & $159 / 191(83.2)$ & $131 / 149(87.9)$ & $28 / 42(66.7)$ & 0.001 & 0.403 & $0.240-0.675$ \\
\hline ICU & 17/191 (8.9) & $12 / 149(8.1)$ & $5 / 42(11.9)$ & ns & 1.383 & $0.628-3.048$ \\
\hline Deceased & 7/191 (3.7) & $0 / 149(0)$ & $7 / 42(16.7)$ & $<0.001$ & 5.257 & $3.902-7.083$ \\
\hline Home & 6/191 (3.1) & $5 / 149(3.4)$ & $1 / 42(2.4)$ & ns & 0.752 & $0.123-4.593$ \\
\hline
\end{tabular}

Abbreviations: RR: relative risk; C.I.: confidence interval; BMI: body-mass index; HBP: high blood pressure; DM: diabetes mellitus; COPD: chronic obstructive pulmonary disease; FEV1: forced expiratory volume in one second; Hypov. syndr.: hypoventilation syndromes; LTOT: long-term oxygen therapy; Smoking: personal history of smoking; Hb: hemoglobin (gr/L); Creat: creatinine (mg/ dL); Bicarb: bicarbonate (mmol/L); Lact: lactic acid (mmol/L); ER: emergency room; CHF: congestive heart failure; RF: respiratory failure due to pulmonary origin; Hospitaliz.: hospitalization ground; ICU: intensive care unit; ns: not significant.

Table 2: General characteristics of the patients. Comparison between survivors and deceased within 30 days from emergency room admission. Quantitative variables.

\begin{tabular}{|c|c|c|c|c|c|c|c|}
\hline \multirow[b]{2}{*}{ Variable } & \multicolumn{2}{|c|}{ All cases } & \multicolumn{2}{|c|}{ Survivors } & \multicolumn{2}{|c|}{ Deceased } & \multirow{2}{*}{$\begin{array}{l}P \\
\text { value }\end{array}$} \\
\hline & n (191) & Value & n (149) & Value & n (42) & Value & \\
\hline \multicolumn{8}{|c|}{ Main objective biomarker } \\
\hline MR-proADM (median) & 191 & $0.57(0.22)$ & 149 & $0.57(0.26)$ & 42 & $0.57(0.14)$ & ns \\
\hline \multicolumn{8}{|c|}{ Personal characteristics } \\
\hline Age (median) & 191 & $79(15)$ & 149 & $77(17)$ & 42 & $83(10)$ & 0.001 \\
\hline BMI (mean) & 173 & $30.508 \pm 7.343$ & 139 & $30.53 \pm 6.975$ & 34 & $30.4 \pm 8.811$ & ns \\
\hline \multicolumn{8}{|l|}{ Vital status variables } \\
\hline Glasgow (median) & 184 & $15(1)$ & 144 & $15(1)$ & 40 & $15(3)$ & 0.006 \\
\hline MAP (median) & 181 & $92(22)$ & 147 & $93(23)$ & 42 & $88(22)$ & ns \\
\hline H.R. (mean) & 181 & $90 \pm 21.462$ & 147 & $89 \pm 21.598$ & 42 & $95 \pm 21.198$ & ns \\
\hline Temp. (median) & 181 & $36.5(0.8)$ & 142 & $36.4(0.8)$ & 39 & $36.6(0.8)$ & ns \\
\hline \multicolumn{8}{|l|}{ Laboratory variables } \\
\hline $\mathrm{pH}$ (mean) & 190 & $7.28 \pm 0.099$ & 148 & $7.28 \pm 0.101$ & 42 & $7.27 \pm 0.093$ & ns \\
\hline $\mathrm{PaCO}_{2}$ (median) & 191 & $60.3(24.34)$ & 149 & $60.3(24.08)$ & 42 & $50.5(25.18)$ & ns \\
\hline $\mathrm{HCO}_{3}^{-}$(media) & 191 & $25.44 \pm 5.954$ & 149 & $25.84 \pm 5.957$ & 42 & $24 \pm 5.785$ & ns \\
\hline $\mathrm{PaO}_{2} / \mathrm{FiO}_{2}$ (mean) & 77 & $213.39 \pm 117.59$ & 65 & $214.31 \pm 122.19$ & 12 & $208.42 \pm 93.03$ & ns \\
\hline Lact. (median) & 182 & $1.7(1.7)$ & 143 & $1.6(1.3)$ & 39 & $2.3(2)$ & ns \\
\hline Hb. (mean) & 191 & $12.57 \pm 2.137$ & 149 & $12.51 \pm 2.160$ & 42 & $12.78 \pm 2.064$ & ns \\
\hline
\end{tabular}




\begin{tabular}{|l|l|l|l|l|l|l|l|}
\hline Leuk. (mean) & 191 & $11.7 \pm 5.391$ & 149 & $11.3 \pm 4.414$ & 42 & $13.1 \pm 7.867$ & $\mathrm{~ns}$ \\
\hline Plat. (median) & 191 & $244(104)$ & 149 & $244(117)$ & 42 & $258(87)$ & $\mathrm{ns}$ \\
\hline Urea (median) & 191 & $55(40)$ & 149 & $54(41)$ & 42 & $65(45)$ & $\mathrm{ns}$ \\
\hline Creat. (median) & 191 & $1.3(0.8)$ & 149 & $1.3(0.7)$ & 42 & $1.5(1.43)$ & $\mathrm{ns}$ \\
\hline $\mathrm{Na}^{+}$(median) & 191 & $139(7)$ & 149 & $139(7)$ & 43 & $139(11)$ & $\mathrm{ns}$ \\
\hline $\mathrm{K}^{+}$(median) & 177 & $4.6(1)$ & 143 & $4.6(0.8)$ & 34 & $4.75(1.3)$ & $\mathrm{ns}$ \\
\hline INR (median) & 191 & $1.09(0.64)$ & 149 & $1.09(0.77)$ & 42 & $1.23(0.45)$ & $\mathrm{ns}$ \\
\hline
\end{tabular}

Abbreviations and measurement units: BMI: body-mass index; MAP: mean arterial pressure (mmHg); H.R.: heart rate (beats per minute); Temp: temperature (degrees Celsius); $\mathrm{PaCO}_{2}$ : arterial carbon dioxide tension $(\mathrm{mmHg}) ; \mathrm{HCO}_{3}:$ : bicarbonatein arterial blood (mmol/L); $\mathrm{PaO}_{2} / \mathrm{FiO}_{2}$ : arterial oxygen tension/fraction of inspired oxygen; Lact.: lactic acid in arterial blood (mmol/L); $\mathrm{Hb}$ :: hemoglobin (gr/L); Leuk.: leukocytes (x1000); Plat.: platelets (x1000); Serum urea (mg/dL); Creat.: serum creatinine (mg/dL); Na+: sodium ion ( $\mathrm{mmol} / \mathrm{L})$; $\mathrm{K}^{+}$: potassium ion ( $\left.\mathrm{mmol} / \mathrm{L}\right)$; INR: international normalized ratio; MR-proADM: mid-regional proadrenomedullin (nmol/L); ns: not significant.

Note: The means are expressed followed by standard deviation and refer to variables with normal distribution while the medians are expressed followed (in parentheses) of the interquartile range and refer to non-parametric variables.

Table 3: General characteristics of the patients according to the concentrations of MR-proADM stratified by quartiles. Qualitative variables.

\begin{tabular}{|c|c|c|c|c|c|}
\hline Variable & $\begin{array}{l}\text { I Quartil }(\mathrm{n}=56) \\
<0.5 \mathrm{nmol} / \mathrm{L}\end{array}$ & $\begin{array}{l}\text { II Quartil }(\mathrm{n}=44) \\
0.51-0.57 \mathrm{nmol} / \mathrm{L}\end{array}$ & $\begin{array}{l}\text { III Quartil }(\mathrm{n}=44) \\
0.571-0.718 \mathrm{nmol} / \mathrm{L}\end{array}$ & $\begin{array}{l}\text { IV Quartil }(n=47) \\
>0.718 \mathrm{nmol} / \mathrm{L}\end{array}$ & P value* \\
\hline \multicolumn{6}{|l|}{ Personal characteristics } \\
\hline Age $>75$ años & $32(27)$ & $33(28)$ & $25(21.2)$ & $28(23.7)$ & ns \\
\hline Gender $=$ male & $31(29.3)$ & $22(23)$ & $26(23)$ & $19(19.4)$ & ns \\
\hline $\mathrm{BMI}>30$ & $29(35.4)$ & $18(22)$ & $16(29.5)$ & $19(23.2)$ & ns \\
\hline Smoking & $33(34)$ & $18(18.6)$ & $24(24.7)$ & $22(22.7)$ & ns \\
\hline \multicolumn{6}{|l|}{ Personal history } \\
\hline HBP & $49(29.5)$ & $41(24.7)$ & $38(22.9)$ & $38(22.9)$ & ns \\
\hline DM & $26(28)$ & $23(24.7)$ & $23(24.7)$ & $21(22.6)$ & ns \\
\hline COPD & $25(32.9)$ & $14(18.4)$ & $19(25)$ & $18(23.7)$ & ns \\
\hline FEV1 $<50 \%$ & $10(22.2)$ & $8(17.8)$ & $13(28.9)$ & $14(31.1)$ & ns \\
\hline Hypov. Syndr. & $13(31.7)$ & $12(29.3)$ & $8(19.5)$ & $8(19.5)$ & ns \\
\hline Heart diseases & $37(29.6)$ & $28(22.4)$ & $31(24.8)$ & $29(23.2)$ & ns \\
\hline Arrhythmias & $23(26.1)$ & $20(22.7)$ & $20(22.7)$ & $25(28.4)$ & ns \\
\hline LTOT & $21(32.8)$ & $8(12.5)$ & $16(25)$ & $19(29.7)$ & ns \\
\hline Domiciliary NIV & $16(34)$ & $11(23.4)$ & $10(21.3)$ & $10(21.3)$ & ns \\
\hline \multicolumn{6}{|l|}{ Laboratory variables } \\
\hline Hemoglobin < $12 \mathrm{gr} / \mathrm{L}$ & $23(29.5)$ & $18(23.1)$ & $18(23.1)$ & $19(24.4)$ & ns \\
\hline Creatinine $>1.5 \mathrm{mg} / \mathrm{dL}$ & $19(23.5)$ & $22(27.2)$ & $16(19.8)$ & $24(29.6)$ & ns \\
\hline Bicarbonate $<22 \mathrm{mmol} / \mathrm{L}$ & $9(16.1)$ & $14(25)$ & $16(28.6)$ & $17(30.4)$ & ns \\
\hline Lactic acid $>2 \mathrm{mmol} / \mathrm{L}$ & $22(28.9)$ & $17(22.4)$ & $18(23.7)$ & $19(25)$ & ns \\
\hline \multicolumn{6}{|l|}{ ER discharge diagnoses } \\
\hline $\mathrm{CHF}$ & $23(25.8)$ & $26(29.2)$ & $22(24.7)$ & $18(20.2)$ & ns \\
\hline RF & $28(34.6)$ & $12(14.8)$ & $16(19.8)$ & $25(30.9)$ & ns \\
\hline Sepsis & $0(0)$ & $2(33.3)$ & $2(33.3)$ & $2(33.3)$ & ns \\
\hline Pneumonía & $2(28.6)$ & $2(28.6)$ & $2(28.6)$ & $1(14.3)$ & ns \\
\hline Others & $3(37.5)$ & $2(25)$ & $2(25)$ & $1(12.5)$ & ns \\
\hline \multicolumn{6}{|l|}{ ER discharge destination } \\
\hline Hospitalization ground & $50(31.1)$ & $40(24.8)$ & $35(21.7)$ & $36(22.4)$ & ns \\
\hline ICU & $2(11.8)$ & $3(17.6)$ & $5(29.4)$ & 7 (41.2) & ns \\
\hline Deceased & $1(14.3)$ & $0(0)$ & $4(57.1)$ & $2(28.6)$ & ns \\
\hline Home & $3(50)$ & $1(16.7)$ & $0(0)$ & $2(33.3)$ & ns \\
\hline
\end{tabular}

"Based on the statistical "ji-square" test.

Abbreviations: BMI: body-mass index; HBP: high blood pressure; DM: diabetes mellitus; COPD: chronic obstructive pulmonary disease; FEV1: forced expiratory volume in one second; Hypov. syndr.: hypoventilation syndromes; LTOT: long-term oxygen therapy; NIV: non-invasive ventilation; ER: emergency room; CHF: chronic heart failure; RF: respiratory failure due to pulmonary origin; ICU: intensive care unit; ns: not significant.

Note: The values in the table correspond to the "n" represented in each group followed by the corresponding percentage.

found in (Table 1) and (Table 2), in which the possible relation between the factors and the dependant variable is also shown "30-day mortality", finding an association $(p<0.05)$ with the variables "Age > 75-years-old",
"Lactic Acid > 2 mmol/L" and Sepsis diagnose.

A statistical analysis was carried out distributing the variable MR-proADM in quartiles, generating 4 groups and the patients main characteristics were studied 
again in order to find out if there were any differences between them (Table 3) and (Table 4), but no differences were found.

In the multivariate analysis only the statistically significant were associated "Age > 75-years-old", and Sepsis diagnose (Table 5, Figure 3), while the "Lactic Acid" and "MR-proADM were not included in the model.

\section{Discussion}

Remember that the medians found from MR-proADM were $0.57 \mathrm{nmol} / \mathrm{L}$ for the survivors and for the deceased, and that there were no significant statistical differences in median MR-proADM for both groups when stratifying the sample.

The ARF, regardless of its cause, has been associated with a very poor prognosis and has been identified as a significant risk factor for mortality which includes from cardiac causes to any other cause in patients with dyspnea [17]. In our survey we have included patients with various diseases which were the source of severe acute dyspnea, such major diseases range from cardiac causes like CHF, Coronary ischemia or ACPE; causes of respiratory origin as exacerbation of COPD or asthma; causes of infectious origin like sepsis or pneumonia; and any other development that could be within the range of the adjunctive spectrum of ARF. The fact that many of these etiologies have demonstrated their expression in the MR-proADM and its reflection on predicting mortality marked our research process; however, in view of the results, we are able to say that we did not find statistically significant differences between the figures for MR-proADM obtained during admission of survivors and deceased patients, neither as a whole nor after stratifying our results according to gender, age or diagnosis group.

Table 4: General characteristics of patients depending on the concentrations of MR-proADM stratified into quartiles. Quantitative variables.

\begin{tabular}{|c|c|c|c|c|c|c|}
\hline Variable & $\mathbf{n}$ & $\begin{array}{l}\text { I Quartil }(n=56) \\
<0.5 \mathrm{nmol} / \mathrm{L}\end{array}$ & $\begin{array}{l}\text { II Quartil }(\mathrm{n}=44) \\
0.51-0.57 \mathrm{nmol} / \mathrm{L}\end{array}$ & $\begin{array}{l}\text { III Quartil }(\mathrm{n}=44) \\
0.571-0.718 \mathrm{nmol} / \mathrm{L}\end{array}$ & $\begin{array}{l}\text { IV Quartil }(n=47) \\
>0.718 \mathrm{nmol} / \mathrm{L}\end{array}$ & P value ${ }^{*}$ \\
\hline \multicolumn{7}{|c|}{ Personal characteristics } \\
\hline Age (median) & 191 & $79(20)$ & $80(12)$ & 77 (15) & $77(11)$ & ns \\
\hline BMI (mean) & 173 & $30.74 \pm 9.01$ & $31.28 \pm 7.14$ & $29.85 \pm 5.73$ & $30.51 \pm 6.66$ & ns \\
\hline \multicolumn{7}{|c|}{ Vital status variables } \\
\hline Glasgow (median) & 184 & $15(3)$ & $15(0)$ & $15(1)$ & $15(2 t)$ & ns \\
\hline MAP (median) & 181 & $94(24)$ & $92(22)$ & $92(19)$ & $91(27)$ & ns \\
\hline H.R. (media) & 181 & $91 \pm 18$ & $90 \pm 25$ & $89 \pm 22$ & $91 \pm 23$ & ns \\
\hline Temp. (median) & 181 & $36.4(0.8)$ & $36.6(0.9)$ & $36.5(1)$ & $36.4(0.7)$ & ns \\
\hline \multicolumn{7}{|c|}{ Laboratory variables } \\
\hline $\mathrm{pH}$ (mean) & 190 & $7.29 \pm 0.1$ & $7.28 \pm 0.09$ & $7.28 \pm 0.1$ & $7.27 \pm 0.11$ & ns \\
\hline $\mathrm{PaCO}_{2}$ (median) & 191 & $66.07(40.03)$ & $57.36(26.4)$ & $54.36(23.56)$ & $62.82(18.5)$ & ns \\
\hline $\mathrm{HCO}_{3}^{-}$(mean) & 191 & $27.7 \pm 6.4$ & $24.5 \pm 4.7$ & $23.9 \pm 5.6$ & $25.1 \pm 6.1$ & ns \\
\hline $\mathrm{PaO}_{2} / \mathrm{FiO}_{2}$ (mean) & 77 & $210 \pm 102$ & $208 \pm 97$ & $211 \pm 118$ & $231 \pm 172$ & ns \\
\hline Lact. (median) & 182 & $1.5(1.5)$ & $1.8(1.7)$ & $1.8(1.7)$ & $1.9(2.1)$ & ns \\
\hline Hb. (mean) & 191 & $12.4 \pm 2.03$ & $12.4 \pm 2.02$ & $12.9 \pm 2.4$ & $12.5 \pm 2.2$ & ns \\
\hline Leuk. (mean) & 191 & $10.8 \pm 4.5$ & $11.7 \pm 4.3$ & $11.8 \pm 4.4$ & $12.7 \pm 7.7$ & ns \\
\hline Plat. (median) & 191 & $251(98)$ & $226(96)$ & $232(99)$ & $262(128)$ & ns \\
\hline Urea (median) & 191 & $49(32)$ & $56(47)$ & $58(45)$ & $61(53)$ & ns \\
\hline Creat. (median) & 191 & $1.1(0.6)$ & $1.5(0.6)$ & $1.3(0.8)$ & $1.5(0.8)$ & ns \\
\hline $\mathrm{Na}^{+}$(median) & 191 & $139(8)$ & $139(7)$ & $138(9)$ & $140(8)$ & ns \\
\hline $\mathrm{K}^{+}$(median) & 177 & $4.6(1.1)$ & $4.6(0.8)$ & $4.6(1.1)$ & $4.5(1.3)$ & ns \\
\hline INR (median) & 191 & $1.1(0.5)$ & $1.1(0.5)$ & $1.1(0.4)$ & $1.2(1)$ & ns \\
\hline
\end{tabular}

Abbreviations and measurement units: BMI: body-mass index; MAP: mean arterial pressure (mmHg); H.R.: heart rate (beats per minute); Temp: temperature (degrees Celsius); $\mathrm{PaCO}_{2}$ : arterial carbon dioxide tension $(\mathrm{mmHg}) ; \mathrm{HCO}_{3}:$ : bicarbonate in arterial blood (mmol/L); $\mathrm{PaO}_{2} / \mathrm{FiO}_{2}$ : arterial oxygen tension/fraction of inspired oxygen; Lact.: lactic acid in arterial blood (mmol/L); $\mathrm{Hb}$.: hemoglobin (gr/L); Leuk.: leukocytes (x1000); Plat.: platelets (x1000); Serum urea (mg/dL); Creat.: serum creatinine (mg/dL); Na ${ }^{+}$: sodium ion (mmol/L); $\mathrm{K}^{+}$: potassium ion ( $\left.\mathrm{mmol} / \mathrm{L}\right)$; INR: international normalized ratio; MR-proADM: mid-regional proadrenomedullin (nmol/L); ns: not significant.

Note: The means are expressed with standard deviation and they make reference to variables with normal distribution while the medians are expressed followed (in parentheses) of the interquartile range and refer to non-parametric variables.

*Comparisons between groups were performed by the ANOVA test for normally distributed variables and Kruskal-Wallis for nonparametric.

Table 5: Multivariate analysis. Variables in the equation as risk prognosis after the analysis by Cox regression.

\begin{tabular}{|l|l|l|l|l|}
\hline \multicolumn{1}{|c|}{ B } & P value & Hazard ratio & C.I. 95\% \\
\hline Step 2 & \multicolumn{5}{|l|}{} \\
\hline Age $>$ 75 años & 1.493 & 0.002 & 4.449 & $1.722-11.497$ \\
\hline Outcome $=$ Sepsis & 1.911 & $<0.001$ & 6.76 & $2.550-17.921$ \\
\hline
\end{tabular}




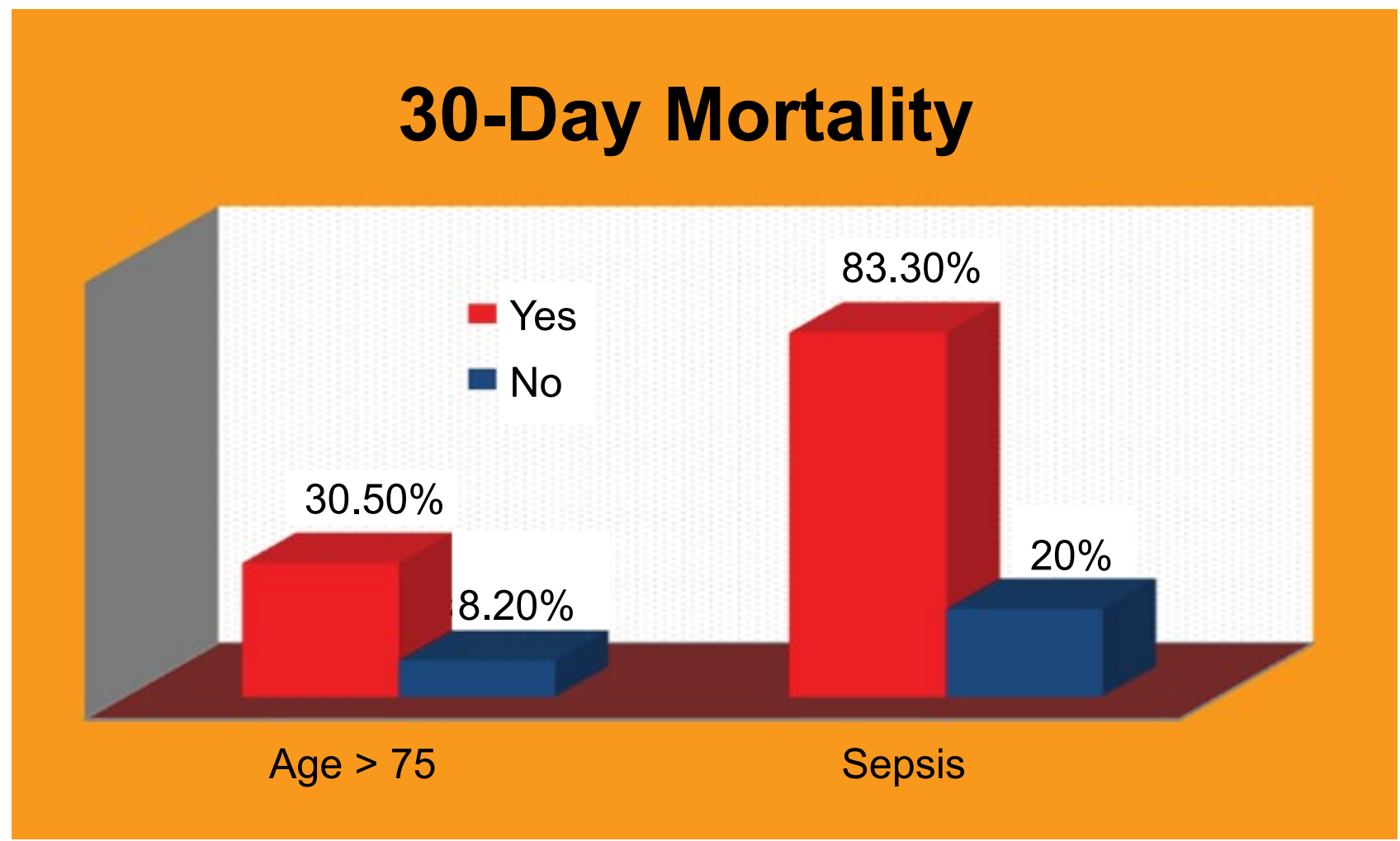

Figure 3: Distribution of the "30-day mortality" for the two variables which remained after Cox regression.

"YES = complies with the condition imposed by the variable. NO = does not comply with the condition imposed by the variable.

The BACH (Biomarkers in Acute Heart Failure) assay included 1,641 patients treated at the emergency room with dyspnea and distributed in 15 hospital facilities, 6 in two European countries and 8 in the US, generating after confirmation, two groups of patients diagnosed with acute dyspnea: those suffering from AHF (34.6\%) and those without it (65.4\%). Among the biomarkers studied, MR-proADM played a key role; in fact, Maisel, et al. published in 2010 in the Journal of the American College of Cardiology [18] the results obtained of the $\mathrm{BACH}$, in it he warns that the MR-proADM income obtained a range between 0.1 and $12.6 \mathrm{nmol} / \mathrm{L}$, with the median of $0.88 \mathrm{nmol} / \mathrm{L}$ (IQR 0.57 to $1.44 \mathrm{nmol} / \mathrm{L}$ ); This is the first major difference with our findings whose median was $0.57 \mathrm{nmol} / \mathrm{L}(\mathrm{IQR}=0.22$ ) covering the range from 0.29 upto $3.19 \mathrm{nmol} / \mathrm{L}$. Another big difference is found in overall mortality, in his results, the survivors at 30 days had $0.739 \mathrm{nmol} / \mathrm{L}$ median against $0.57 \mathrm{nmol} / \mathrm{L}$ for ours. Continuing with inter assay differences, there were also differences within cardiovascular mortality at 30 days with a median of $0.790 \mathrm{nmol} / \mathrm{L}$ for them and $0.54 \mathrm{nmol} / \mathrm{L}$ for us.

As a matter of fact, we have not found similarities between our MR-proADM results and those from other consulted surveys, for instance, Herrero Puente, et al. in a pilot survey on the usefulness of MR-proADM in the assessment o dyspnea from respiratory origin in the ER [19] obtained an average figure of $1 \mathrm{nmol} / \mathrm{L}$ (range between 0.23 and 2.69) while our average was 0.67 $\mathrm{nmol} / \mathrm{L}$ (range between 0.29 and 3.19). Dieplinger, et al. [20] published his results on patients with dyspnea in the ER and finds that the survivors have a median of MR-proADM $0.69 \mathrm{nmol} / \mathrm{L}$ compared to 1.14 for those who deceased, also showing its discrepancies with our data. Another survey published by Courtais, et al., on community-acquired pneumonia [21], shows median MR-proADM of $1.035 \mathrm{nmol} / \mathrm{L}$ for survivors, admitted for this reason, at 30 days versus $3.017 \mathrm{nmol} / \mathrm{L}$ for the ones deceased $(p=0.003)$; our results in this condition and for the same monitoring time show $0.53 \mathrm{nmol} / \mathrm{L}$ for survivors and $0.62 \mathrm{nmol} / \mathrm{L}$ for the deceased $(p=0.614)$. Finally, with respects to the diagnosis of "sepsis" we did not find matches with other authors; our results in this subgroup reflect a median of $0.66 \mathrm{nmol} / \mathrm{L}$ with a range between 0.53 and 3.19 for the deceased, which we will recall that amounted to 83.3\%; however Christ-Crain, et al. [22] found that his patients with severe sepsis who did not survive on admission were $8.5 \mathrm{nmol} / \mathrm{L}$ median (range 0.8 to 21) compared to $1.7 \mathrm{nmol} / \mathrm{L}$ of which overcame the crisis; or Suberviola, et al. [23] who obtained figures of $5.0 \mathrm{nmol} / \mathrm{L}$ for patients deceased during hospitalization vs. $1.7 \mathrm{nmol} / \mathrm{L}$ for survivors.

It is clear therefore, that in our survey no evidence is related between the concentrations of MR-proADM and mortality, as it was objectified in the other described surveys. The first striking difference between our survey and the rest is the type of patients; ours had severe ARF, while in others there was a greater amalgam of them, and therefore, less severe than ours; thus, it is possible that the MR-proADM is more effective diffe- 
rentiating patients between degrees of severities. The second difference could be due to the fact that we have included a wide range of precipitating causes of acute dyspnea with diagnostic groups which comprehend entities, that even though having common peculiarities, indeed with no consistent physiopathology and perhaps too small to stratify in the analysis.

One of the major limitations of the survey was due to the heterogeneity of the target population in terms of clinical diagnoses (various causes of dyspnea), which surely prevents that there is a correlation between the concentrations of biomarkers and mortality; in addition, cut-off points can vary depending on the target population (emergencies, critical patient, post-surgery) and depending on the clinical diagnosis, as well as in accordance of clinical diagnosis; also the single survey nature of the survey has limited the " $n$ " analyzed both as global and as groups, which we believe has been very important to find significant differences in the stratification, but not to respond to the main objective which referred to patients treated with NIV globally. With respect to plasma sample's freezing, the recommendations from the International Agency for Research on Cancer of the World Health Organization were followed, by what we believe that this process could not have interfered with the results, nor the chosen analysis technique (ELISA), which has demonstrated its high sensitivity for the determination of proteins and hormones and is internationally guaranteed for these procedures. Either way, we believe that wider new surveys with selected patient groups would be required.

Among the prognosis of failure for NIV, guides do not include age [24,25], however, it seems logical that age can be a factor that worsens the prognosis of patients undergoing NIV. In our survey, when we group 75-years-old patients and compare their 30-day mortality with the below that age, we found statistically significant differences in $\mathrm{HR}=4.449$. Other authors like Echave-Sustaeta have also found this association [26]; therefore it is important to consider this variable were not due to being obvious must not it be forgotten.

$3.1 \%$ of the cases included in our survey were diagnosed with sepsis. Of these, we had a fairly high percentage of deaths, $83.3 \%$ especially if compared to other hospital-based surveys; a multicenter survey in Spain reported $40 \%$ [27]; Another survey in the Madrid Region (Spain) found 33\% mortality [28], and other countries like France and Australia published figures between $35 \%$ and $47 \%$ $[29,30]$. This can be explained, however, due to the fact that they conducted the survey on the global sepsis mortality, while we have focused only on those patients who were treated with NIV during admission, regardless of whatever the etiology of sepsis was, which should explain the significant differences found. It is important to point out that all deaths from sepsis occurred during hospitalization and within the first 30 days from admission.

\section{Conclusions}

MR-proADM in our survey, and taking into account the constraints already referred, not predict the 30-day mortality since admission to the ER for the patients who require treatment with NIV, regardless of the precipitant pathology.

Other indicators such as age $>75$-years-old and the diagnosis of sepsis should be taken into account to establish the risk.

\section{Conflict of Interest}

The authors declare that there is no conflict of interest regarding the publication of this paper.

The submitting author takes responsibility for the paper and is responsible for all the other coauthors which ensure that the paper has not been published elsewhere and is not currently under consideration by another journal published by Hindawi or any other publisher; moreover, the article's publication has been approved by all them, and they allow editing of the paper for readability. Finally, the authors declare that there is no conflict of interest regarding the publication of this paper.

\section{References}

1. Levy MM (2011) Preface: Biomarkers in Critical Illness. Crit Care Clin 27: xiii-xv.

2. Kitamura K, Kangawa K, Kawamoto M, Ichiki Y, Nakamura S, et al. (2012) Adrenomedullin: a novel hypotensive peptide isolated from human pheochromocytoma. Biochem Biophys Res Commun 425: 548-555.

3. Beltowski J, Jamroz A (2004) Adrenomedullin - What do we know 10 years since its discovery? Pol J Pharmacol 56: 5-27.

4. Bunton DC, Petrie MC, Hillier C, Johnston F, McMurray JJ (2004) The clinical relevance of adrenomedullin: a promising profile? Pharmacology \& Therapeutics 103: 179-201.

5. Von Haehling S, Filippatos GS, Papassotiriou J, Cicoira M, Jankowska EA, et al. (2010) Mid-regional pro-adrenomedullin as a novel predictor of mortality in patients with chronic heart failure. Eur J Heart Fail 12: 484-491.

6. Khan SQ, O'Brien RJ, Struck J, Quinn P, Morgenthaler N, et al. (2007) Prognostic value of midregional pro-adrenomedullin in patients with acute myocardial infarction. J Am Coll Cardiol 49: 1525-1532.

7. Morgenthaler NG, Struck J, Alonso C, Bergmann A (2005) Measurement of midregional proadrenomedullin in plasma with an immunoluminometric assay. Clin Chem 51: 18231829.

8. Yoshizawa T, Sakurai T, Kamiyoshi A, Ichikawa-Shindo Y, Kawate $\mathrm{H}$, et al. (2013) Novel regulation of cardiac metabolism and homeostasis by the adrenomedullin-receptor activity-modifying protein 2 system. Hypertension 61: 341-351.

9. Tang WHW (2010) Contemporary Challenges in Translating Biomarker Evidence Into Clinical Practice. J Am Coll Cardiol 55: 2077-2079.

10. Potocki M, Breidthardt T, Reichlin T, Morgenthaler NG, Bergmann A, et al. (2009) Midregional pro-Adrenomedullin 
in addition to b-type natriuretic peptides in the risk stratification of patients with acute dyspnea: an observational study. Crit Care 13: 122.

11. Dhillon OS, Khan SQ, Narayan HK, Ng KH, Struck J, et al. (2010) Prognostic value of mid-regional pro-adrenomedullin levels taken on admission and discharge in non-ST-elevation myocardial infarction: the LAMP (Leicester Acute Myocardial Infarction Peptide) II study. J Am Coll Cardiol 56: 125-133.

12. Pezzilli R, Barassi A, Pigna A, Morselli-Labate AM, Imbrogno A, et al. (2012) Time course of proadrenomedullin in the early phase of septic shock. A comparative study with other proinflammatory proteins. Panminerva Med 54: 211-217.

13. Wang RL, Kang FX (2010) Prediction about severity and outcome of sepsis by pro-atrial natriuretic peptide and pro-adrenomedullin. Chin J Traumatol 13: 152-157.

14. Kruger S, Ewig S, Giersdorf S, Hartmann O, Suttorp N, et al. (2010) Cardiovascular and inflammatory biomarkers to predict short- and long-term survival in community-acquired pneumonia: Results from the German Competence Network, CAPNETZ. Am J Respir Crit Care Med 182: 1426-1434.

15. Meng DQ, Li XJ, Song XY, Xin JB, Yang WB (2014) Diagnostic and prognostic value of plasma adrenomedullin in COPD exacerbation. Respir Care 59: 1542-1549.

16. Mas A, Masip J (2014) Noninvasive ventilation in acute respiratory failure. Int J Chron Obstruct Pulmon Dis 9: 837-852.

17. Abidov A, Rozanski A, Hachamovitch R, Hayes SW, Aboul-Enein F, et al. (2005) Prognostic Significance of Dyspnea in Patients Referred for Cardiac Stress Testing. New England Journal of Medicine 353: 1889-1898.

18. Maisel A, Mueller C, Nowak R, Peacock WF, Landsberg JW, et al. (2010) Mid-Region Pro-Hormone Markers for Diagnosis and Prognosis in Acute Dyspnea Results From the BACH (Biomarkers in Acute Heart Failure) Trial. J Am Coll Cardiol 55: 2062-2076.

19. Herrero Puente P, Fernandez Garcia D, Gil Roman JJ, Prieto Garcia B, Vazquez Alvarez J, et al. (2012) Midregional proadrenomedullin plasma concentration in emergency patients with dyspnea due to respiratory disease: a pilot study. Emergencias 24: 357-365.

20. Dieplinger B, Gegenhuber A, Kaar G, Poelz W, Haltmayer $M$, et al. (2010) Prognostic value of established and novel biomarkers in patients with shortness of breath attending an emergency department. Clin Biochem 43: 714-719.
21. Courtais C, Kuster N, Dupuy AM, Folschveiller M, Jreige R, et al. (2013) Proadrenomedullin, a useful tool for risk stratification in high Pneumonia Severity Index score community acquired pneumonia. Am J Emerg Med 31: 215-221.

22. Christ-Crain M, Morgenthaler NG, Struck J, Harbarth S, Bergmann A, et al. (2005) Mid-regional pro-adrenomedullin as a prognostic marker in sepsis: an observational study. Crit Care 9: 816-824.

23. Suberviola B, Castellanos-Ortega A, Llorca J, Ortiz F, Iglesias D, et al. (2012) Prognostic value of proadrenomedullin in severe sepsis and septic shock patients with community-acquired pneumonia. Swiss Med Wkly 142: 13542.

24. Ayuso Baptista F, Jimenez Moral G, Javier Fonseca del Pozo $F$ (2009) Management of acute respiratory failure with noninvasive ventilation in the emergency department. Emergencias 21: 189-202.

25. Keenan SP, Sinuff T, Burns KEA, Muscedere J, Kutsogiannis J, et al. (2011) Clinical practice guidelines for the use of noninvasive positive-pressure ventilation and noninvasive continuous positive airway pressure in the acute care setting. CMAJ 183: E195-E214.

26. Echave-Sustaeta J, Comeche Casanova L, García Luján R, Sayas Catalan J, Gómez de la Cámara A, et al. (2010) Pronóstico tras una agudización grave de la EPOC tratada con ventilación mecánica no invasiva. Archivos de Bronconeumología 46: 405-410.

27. Nin Vaeza N, Lorente Balanza JA, Ortiz-Leyba C, Valenzuela Sánchez F, Baigorri González F, et al. (2005) Estudio multicéntrico sobre la asociación entre variables relacionadas con la resucitación y la mortalidad en la sepsis grave. Medicina Intensiva 29: 212-218.

28. Iñigo J, Sendra JM, Díaz R, Bouza C, Sarría-Santamera A (2006) Epidemiología y costes de la sepsis grave en Madrid. Estudio de altas hospitalarias. Medicina Intensiva 30: 197-203.

29. Brun-Buisson C (2006) Épidémiologie des états septiques graves. La Presse Médicale 35: 513-520.

30. Peake SL, Bailey M, Bellomo R, Cameron PA, Cross A, et al. (2009) Australasian resuscitation of sepsis evaluation (ARISE): A multi-centre, prospective, inception cohort study. Resuscitation 80: 811-818. 